\title{
Network-coding-based Cooperative V2V Communication in Vehicular Cloud Networks
}

\author{
Rui Chen*, Weijun Xing*, Chao Wang*†, Ping Wang*, Fuqiang Liu*, and Yusheng $\mathrm{Ji}^{\ddagger}$ \\ ${ }^{*}$ Department of Information and Communication Engineering, Tongji University, Shanghai, China \\ ${ }^{\dagger}$ Department of Computer Science, University of Exeter, Exeter, U.K. \\ ${ }^{\ddagger}$ National Institute of Informatics, Graduate University for Advanced Studies, Japan
}

\begin{abstract}
We investigate the potential of applying cooperative relaying and network coding techniques to support vehicle-to-vehicle (V2V) communication in vehicular cloud networks ( $\mathrm{VCN})$. A reuse-mode MIMO content distribution system with multiple sources, multiple relays, and multiple destinations under Nakagami-m fading is considered. We apply a class of finite field network codes in the relays to achieve high spatial diversity in an efficient manner and derive the system communication error probability that the destinations fail to recover the desired source messages. The results show that our method can improve the performance over conventional data transmission solutions.
\end{abstract}

Index Terms

Vehicular cloud networks, network coding, cooperative relaying.

\section{INTRODUCTION}

In the past decades, the ever-increasing numbers of vehicles have caused serious problems, such as congestion, accidents and pollution, all over the world. There is a strong desire for an efficient, safe, and clean road traffic system. The concept of intelligent transportation system (ITS) enabled by information and communication technology (ICT) has been accepted as the most promising solution [1]. As a key element in ITS, "smart vehicles" equipped with advanced on-board sensing and computation devices are designed to help reducing accidents caused by human errors, through the advance driving assistance system (ADAS) or even self-driving functions. Google's self-driving car and Baidu's Apollo platform are examples of autonomous driving vehicle and developing platform prototypes.

One issue with most today's smart vehicle prototypes is that they normally work individually. Even though they have advanced sensors, their sensing range and reliability are relatively limited in complex driving conditions. Allowing vehicles to interact through wireless communications is seen as an effective solution. Since the application scenario is very different from traditional mobile Internet/wireless LAN, a number of wireless technologies dedicated for establishing the Internet of vehicles or V2X (vehicle to everything) communications have been developed in recent years, including e.g., the IEEE 802.11p Dedicated Short Range Communication (DSRC) technology and some LTE-based solutions [2]. More importantly, the current 5G research campaign also considers vehicular communication environment as a key application scenario [3].

With the support of $5 \mathrm{G}$ and other advanced V2X technologies, the data exchanged among vehicles and road-side units would not be limited to conventional small-size heart-beat or event-driven messages. Sharing content-rich sensing data will be feasible, which triggers a promising concept Vehicular Cloud Network (VCN) [4], [5]. VCN stems from the idea of cloud computing and is envisioned as a framework where multiple vehicles share their sensing, communication, computation, and storage resources to realize functions that each individual vehicle cannot. For example, sharing sensing data allows each vehicle to access the others' sensing resources to extend its own ability of perceiving the surrounding environment. Sharing communication resources (e.g., bandwidth and power) potentially enhances the data exchange capability within the network.

Two example application scenarios of VCN are shown in Fig. 1. The left hand side (LHS) illustrates a VCN with five vehicles conducting cooperative objective detection in complicated driving conditions such as road intersections [6]. $\mathrm{V}_{1}$ and $\mathrm{V}_{2}$ have advantageous locations to obtain accurate sensing results regarding their surrounding environment, e.g., the existence, position and movement of obstacles near the intersection corner. Sharing their sensing data with those who relatively far away from the traffic scene (i.e., $V_{4}$ and $V_{5}$ ) enables greatly improved sensing range. The right hand side (RHS) of Fig. 1 shows a VCN formed by six autonomous vehicles operating in a cooperative driving scenario [7]. Now $V_{1}$ and $V_{6}$ act as sensing information sources and desire to distribute their data to $V_{2}$ and $V_{5}$. These illustrate the concept of sensing resource sharing.

However, due to fast changing network topology and complicated signal propagation environment, the quality of message sharing is often hard to guarantee. In this case, cooperative relaying can serve to significantly improve performance [8]. For example, in the LHS scenario of Fig. 1, $\mathrm{V}_{3}$ shares its own communication resources with others and serves as a relay to guarantee the sensing data to be delivered from $V_{1}$ and $V_{2}$ to $V_{4}$ and $V_{5}$ with low probability of failure. In the RHS scenario, the message sharing can be helped by $\mathrm{V}_{3}$ and $\mathrm{V}_{4}$ acting as relays.

The conventional relay forwarding operation is to directly repeat received signals. In multi-source scenarios, such a repetitioncoding based relaying strategy does not efficiently use the available channel resources. In this paper, we study applying 


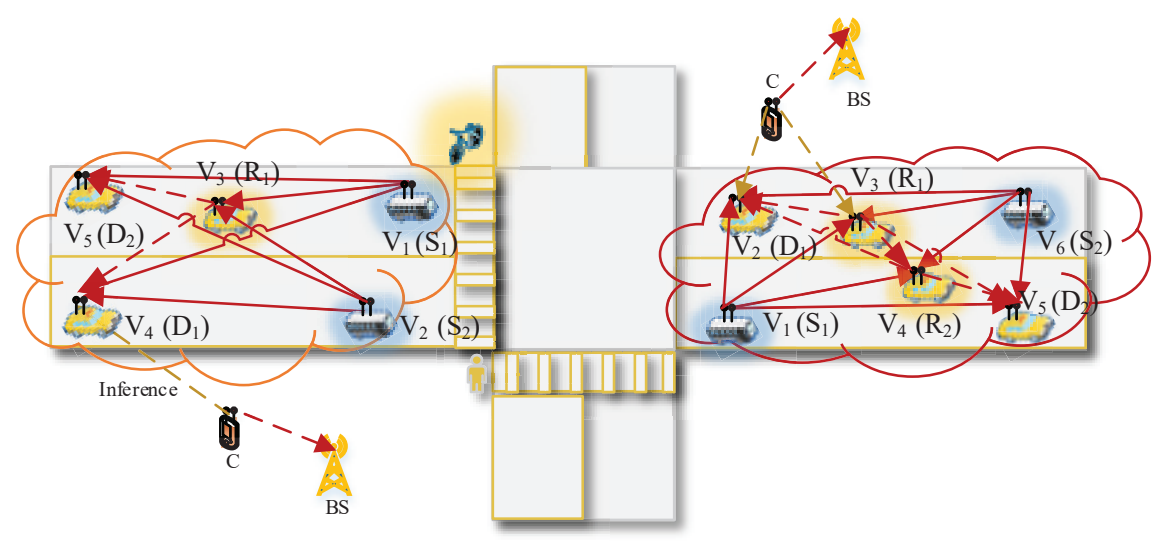

Fig. 1. System model.

network coding techniques to handle this issue. Specifically, we consider a reuse-mode multi-source multi-relay multi-destination cooperative MIMO V2V communication scenario in VCN. A class of maximum distance separable finite field network codes (MDS-FFNC) [9]-[11] is applied in the relays to attain high spatial diversity in an efficient fashion. We derive the system communication error probability, in a Nakagami-m fading environment. It is shown that our method can improve performance over conventional direct transmission and repetition-coding based relaying schemes.

\section{SYSTEM MODEL}

We consider a VCN scenario with a total of $U$ member vehicles $\mathrm{V}_{1}, \cdots, \mathrm{V}_{U}$, among which $M$ vehicles (denoted as sources $\mathrm{S}_{1}, \cdots, \mathrm{S}_{M}$ ) have attained some sensing data from their own sensors, represented respectively by independent messages $I_{\mathrm{S}_{1}}, \cdots, I_{\mathrm{S}_{M}}$, and intend to share them (i.e., sensing resource sharing) with $N$ other members (denoted as destinations $\mathrm{D}_{1}$, $\cdots, \mathrm{D}_{N}$ ). To ensure the quality of the desired message sharing, $K$ vehicles (denoted as relays $\mathrm{R}_{1}, \cdots, \mathrm{R}_{K}$ ) can serve as cooperative relays (i.e., communication resource sharing). Practical applications abstracted by such a multi-source multi-relay multi-destination content distribution network are illustrated in Fig. 1.

The set of the $M$ source messages to be shared within the VCN is denoted as $\mathcal{I}=\left\{I_{\mathrm{S}_{1}}, \cdots, I_{\mathrm{S}_{M}}\right\}$. We consider the case that every destination $\mathrm{D}_{i}$ is interested in attaining the whole source message set $\mathcal{I}$ in order to reinforce its own sensing capability. Therefore, the communication in the considered VCN is successful only if all $\mathrm{D}_{1}, \cdots, \mathrm{D}_{N}$ correctly recover $\mathcal{I}$. Otherwise, if any of them is not capable of completely recovering $\mathcal{I}$, a system communication error is reported.

In addition, in order to efficiently utilize available channel resources, we consider that the V2V transmissions in our VCN can be potentially operated in a reuse mode. In other words, all the aforementioned message delivery process can coexist with the uplink transmission of a certain cellular user, denoted as C. A proper cellular user scheduling strategy is assumed such as that $\mathrm{C}$ is chosen to be close to its serving base station. The V2V transmission power is limited, since message sharing in the considered VCN occurs locally in a limited area, to avoid introducing intolerable interference to the base station. But the reception of each member in the VCN may experience interference from $\mathrm{C}$.

Every node $a$ in the considered system can potentially have $A_{a} \geq 1$ antennas. All message transmissions are conducted in a narrow-band Nakagami-m slow fading environment. The channel fading matrix between transmitting node $a$ and receiving node $b$ is denoted by an $A_{b} \times A_{a}$ matrix $\mathbf{H}_{b, a}$. The element on the $j$ th $\left(j \in\left\{1, \cdots, A_{b}\right\}\right)$ row and $i$ th column $\left(i \in\left\{1, \cdots, A_{a}\right\}\right)$ of $\mathbf{H}_{b, a}$, denoted by $h_{b^{j}, a^{i}}$, represents the fading coefficient between the $i$ th antenna of $a$ and $j$ th antenna of $b$. All the coefficients in $\mathbf{H}_{b, a}$ are modeled by independently and identically distributed (i.i.d.) random variables with absolute values following a Nakagami distribution with parameters $m_{b, a}$ (Nakagami-m fading parameter) and $\hat{\Omega}_{b, a}$ (expected channel power gain) i.e., $\left|h_{b^{j}, a^{i}}\right| \sim \operatorname{Nakagami}\left(m_{b, a}, \hat{\Omega}_{b, a}\right)$. This means that the channel power gain $\left|h_{b^{j}, a^{i}}\right|^{2}$ follows a Gamma distribution with shape parameter $\alpha_{b, a}=m_{b, a}$ and rate parameter $\beta_{b, a}=\frac{m_{b, a}}{\hat{\Omega}_{b, a}}[12]$.

The message exchange in the considered VCN is conducted in a slotted fashion. Each message is encoded using a capacityachieving Gaussian random codeword, so that focus of performance analysis can be mainly put on the impact of channel variation. Every codeword is transmitted using a unit-bandwidth time-division-multiple-access (TDMA) time slot. The channel fading coefficients remain unchanged during the whole period of transmission and are known only at the associated receivers. Since transmitter-side channel knowledge is not available, dynamic power control and rate adaptation are not performed. Therefore, all the messages $I_{\mathrm{S}_{1}}, \cdots, I_{\mathrm{S}_{M}}$ have the same data rate $R$ bits per codeword. A transmitter $a$ evenly spreads it power $P_{a}$ across its $A_{a}$ antennas and applies orthogonal space-time block coding (OSTBC) to broadcast its message.

In the next section, we will elaborate the message transmission process. 


\section{Message tRAnSmission PROCESS}

The complete transmission of the message set $\mathcal{I}$ from the $M$ sources to the $N$ destinations, through the help of $K$ relays, is carried out using $M+K$ individual time slots, each of which is allocated to a transmitting vehicle. Instead of demanding the relays to simply repeat the source messages, we consider applying MDS-FFNC [9]-[11] at the relays. In particular, each relay $\mathrm{R}_{k}(k \in\{1, \cdots, K\})$ is assigned with a set of MDS-FFNC coding coefficients $\omega_{k}^{[1]}, \cdots, \omega_{k}^{[M]}$. All the coding coefficients within the network are properly constructed in advance to guarantee the transfer matrix $\mathbf{G}=\left[\mathbf{I}_{M} \mathbf{W}\right]^{T}$ to be non-singular, where $\mathbf{I}_{M}$ is an $M \times M$ identity matrix and $\mathbf{W}$ is the $M \times K$ coefficient matrix whose $k$ th column elements are $\omega_{k}^{[1]}, \ldots$, $\omega_{k}^{[M]}$. Therefore, any $M$ elements of the output of the encoding process $\mathbf{G} \cdot\left[\begin{array}{lll}I_{\mathrm{S}_{1}} & \cdots & I_{\mathrm{S}_{M}}\end{array}\right]^{T}$, where summations are taken in a certain finite field, are sufficient to recover $I_{\mathrm{S}_{1}}, \cdots, I_{\mathrm{S}_{M}}$. Using these coefficients, if relay $\mathrm{R}_{k}$ correctly attains the complete message set $\mathcal{I}$, it can re-encode the source messages to a new message using $I_{\mathrm{R}_{k}}=\sum_{i=1}^{M} \omega_{k}^{[i]} I_{\mathrm{S}_{i}}$. Knowing any $M$ messages among $I_{\mathrm{S}_{1}}, \cdots, I_{\mathrm{S}_{M}}, I_{\mathrm{R}_{1}}, \cdots, I_{\mathrm{R}_{K}}$ is sufficient to recover $\mathcal{I}$.

The detailed message transmission process can be described as follows. The first $M$ time slots are allocated to $\mathrm{S}_{1}, \cdots$, $\mathrm{S}_{M}$ (i.e., during the slot $i(i \in\{1, \cdots, M\})$, the source $\mathrm{S}_{i}$ broadcasts its message $I_{\mathrm{S}_{i}}$ to all relays and destinations). After all sources complete transmission, the next $K$ time slots are reserved for the relays $\mathrm{R}_{1}, \cdots, \mathrm{R}_{K}$ respectively. All the relays also listen to each other in order to recover the source messages. During time slot $M+k$, if $\mathrm{R}_{k}$ obtains all the messages in $\mathcal{I}$ from its received signals in the previous $M+k-1$ time slots (i.e., it can correctly recover at least $M$ messages among $\left.I_{\mathrm{S}_{1}}, \cdots, I_{\mathrm{S}_{M}}, I_{\mathrm{R}_{1}}, \cdots, I_{\mathrm{R}_{k-1}}\right)$, it re-encodes the source messages into $I_{\mathrm{R}_{k}}$ and forwards it to other relays and the destinations. Otherwise, $\mathrm{R}_{k}$ keeps silent.

Clearly, during each of the $M+K$ time slots, if a transmitting vehicle $a\left(a \in\left\{\mathrm{S}_{1}, \cdots, \mathrm{S}_{M}, \mathrm{R}_{1}, \cdots, \mathrm{R}_{K}\right\}\right)$ is actually activated as being scheduled, the received signal at any receiving vehicle $b\left(b \in\left\{\mathrm{R}_{1}, \cdots, \mathrm{R}_{K}, \mathrm{D}_{1}, \cdots, \mathrm{D}_{N}\right\}\right)$ can be expressed as the following general form:

$$
\mathbf{y}_{b, a}=\sqrt{\frac{P_{a}}{A_{a}}} \mathbf{H}_{b, a} \mathbf{x}_{a}+\sqrt{\frac{P_{C}}{A_{C}}} \mathbf{H}_{b, C} \mathbf{x}_{C}+\mathbf{n}_{b}
$$

where $\mathbf{y}_{b, a}$ is the $A_{b}$-dimensional received signal, $\mathbf{x}_{a}$ is the transmitted signal from $a, \mathbf{x}_{C}$ denotes the co-channel interference from cellular user $C$, and $\mathbf{n}_{b}$ represents additive white Gaussian noise with total power $N_{0}$.

Since each vehicle uses OSTBC to transmit its message in the VCN, the received signal-to-interference-plus-noise-ratio (SINR) can be expressed as [13]

$$
\gamma_{b, a}=\frac{\left\|\mathbf{H}_{b, a}\right\|_{F}^{2} \cdot \rho_{a}}{\left\|\mathbf{H}_{b, C}\right\|_{F}^{2} \cdot \rho_{C}+1}
$$

where $\|\cdot\|_{F}$ represents the Frobenius norm, and $\rho_{a}=\frac{P_{a}}{A_{a} N_{0}}$ and $\rho_{C}=\frac{P_{C}}{A_{C} N_{0}}$ represent transmitter-side signal-to-noise ratio (SNR).

As we mentioned earlier, The probability of occurring system communication error event can be expressed as

$$
P_{\text {err }}=P_{r}\left\{\mathrm{D}_{i} \text { cannot recover } \mathcal{I}, \exists i \in\{1, \cdots, N\}\right\} .
$$

We will provide the method to derive $P_{\text {err }}$ in the following section.

\section{SYSTEM ERROR PERFORMANCE ANALYSIS}

Due to the facts that the probability of activation of each relay depends on the other relays that are scheduled before it, and that the decoding events at different destinations are related (in fact, they are conditionally independent given the decoding set of the relays), the derivation of $P_{\text {err }}$ is actually quite involved. In this paper, we decouple the calculation of $P_{\text {err }}$ into three elementary probability expressions that would be easier to tackle.

The first is the link decoding probability $\mathcal{P}_{b, a}^{L}$ that, during each time slot, a receiving vehicle $b$ correctly decodes the transmit signal of the activated vehicle $a$. We consider Gaussian random coding so that this probability can be found by the probability that the SINR expressed by (2) is larger than the message data rate $R$, i.e., $\mathcal{P}_{b, a}^{L}=P_{r}\left\{\log _{2}\left(1+\gamma_{b, a}\right)>R\right\}$.

The second elementary probability is the probability that a particular set of activated relays occurs. Let us use $\Delta=$ $\left[\delta_{1}, \cdots, \delta_{K}\right]$ to denote the activation behaviors of the $K$ relays after the scheduled $M+K$ time slots, where $\delta_{k}=1$ denotes $\mathrm{R}_{k}$ is able to recover $\mathcal{I}$ from its received signals and $\delta_{k}=0$ denotes the opposite situation. The second elementary probability is denoted by $\mathcal{P}_{\Delta}$.

The third, $\mathcal{P}_{\mathrm{D}_{n} \mid \Delta}(n \in\{1, \cdots, N\})$, is the conditional probability that the destination $\mathrm{D}_{n}$ can fully recover all the source messages given a particular relay activation behavior $\Delta$. Since the destinations' decoding capabilities are conditionally independent, the system's error probability $P_{\text {err }}$ can be calculated by

$$
P_{\text {err }}=1-\sum_{\Delta}\left(\mathcal{P}_{\Delta} \prod_{n=1}^{N} \mathcal{P}_{\mathrm{D}_{n} \mid \Delta}\right),
$$

where the summation is taken for all $\delta_{k} \in\{0,1\}, k \in\{1, \cdots, K\}$, and both $\mathcal{P}_{\Delta}$ and $\mathcal{P}_{\mathrm{D}_{n} \mid \Delta}$ are functions of $\mathcal{P}_{b, a}^{L}$. 
1) Link decoding probability $\mathcal{P}_{b, a}^{L}$ :

The link decoding probability $\mathcal{P}_{b, a}^{L}$ can also be written as $P_{r}\left\{\gamma_{b, a}>2^{R}-1\right\}$. To simplify presentation, we define equivalent received SNR $\hat{\gamma}_{b, a}=\left\|\mathbf{H}_{b, a}\right\|_{F}^{2} \cdot \rho_{a}, \hat{\gamma}_{b, C}=\left\|\mathbf{H}_{b, C}\right\|_{F}^{2} \cdot \rho_{C}$, and $\gamma_{t h}=2^{R}-1$. Now $\mathcal{P}_{b, a}^{L}=P_{r}\left\{\frac{\hat{\gamma}_{b, a}}{\hat{\gamma}_{b, C}+1}>\gamma_{t h}\right\}$.

It is easy to see that $\left\|\mathbf{H}_{b, a}\right\|_{F}^{2}$ (resp. $\left\|\mathbf{H}_{b, C}\right\|_{F}^{2}$ ) is the sum of $A_{b} \times A_{a}$ (resp. $A_{b} \times A_{C}$ ) i.i.d. Gamma random variables with shape parameter $m_{b, a}$ and rate parameter $\frac{m_{b, a}}{\hat{\Omega}_{b, a}}$ (resp. $m_{b, C}$ and $\frac{m_{b, C}}{\hat{\Omega}_{b, C}}$ ) [13]. The sum of $n$ i.i.d. Gamma random variables with shape parameter $\alpha$ and rate parameter $\beta$ is Gamma distributed with parameters $n \alpha$ and $\beta$. Thus the pdf of $\hat{\gamma}_{b, a}$ is

$$
f_{\hat{\gamma}_{b, a}}(x)=\left(\frac{m_{b, a}}{\rho_{a} \hat{\Omega}_{b, a}}\right)^{m_{b, a} A_{b} A_{a}} \cdot \frac{x^{m_{b, a} A_{b} A_{a}-1}}{\Gamma\left(m_{b, a} A_{b} A_{a}\right)} \cdot \exp \left(-\frac{m_{b, a} x}{\rho_{a} \hat{\Omega}_{b, a}}\right) .
$$

The pdf of $\hat{\gamma}_{b, C}$ can be expressed similarly, by replacing $m_{b, a}, A_{a}, \hat{\Omega}_{b, a}, \rho_{a}$ with $m_{b, C}, A_{C}, \hat{\Omega}_{b, C}, \rho_{C}$ respectively. As a result, we can derive $\mathcal{P}_{b, a}^{L}$ as

$$
\mathcal{P}_{b, a}^{L}=\left\{\frac{\hat{\gamma}_{b, a}}{\hat{\gamma}_{b, C}+1} \geq \gamma_{t h}\right\}=\int_{0}^{\infty} P_{r}\left\{\hat{\gamma}_{b, a} \geq \gamma_{t h}(x+1)\right\} f_{\hat{\gamma}_{b, C}}(x) d x .
$$

Denote $\Omega_{b, a}=\rho_{a} \hat{\Omega}_{b, a}$ and $\Omega_{b, C}=\rho_{C} \hat{\Omega}_{b, C}$. Using the CDF of Gamma distribution, we have

$$
P_{r}\left\{\hat{\gamma}_{b, a} \geq \gamma_{t h}(x+1)\right\}=\exp \left(-\frac{m_{b, a} \gamma_{t h}(x+1)}{\Omega_{b, a}}\right) \sum_{k=0}^{m_{b, a} A_{b} A_{a}-1} \frac{1}{k !}\left(\frac{m_{b, a} \gamma_{t h}}{\Omega_{b, a}}(x+1)\right)^{k} .
$$

Now we can substitute (5) and (7) into (6) and take integration

$$
\begin{aligned}
\mathcal{P}_{b, a}^{L}= & \exp \left(-\frac{m_{b, a} \gamma_{t h}}{\Omega_{b, a}}\right)\left(\frac{m_{b, C}}{\Omega_{b, C}}\right)^{A_{b} A_{C} m_{b, C}} \frac{1}{\Gamma\left(A_{b} A_{c} m_{b, C}\right)} \sum_{k=0}^{A_{b} A_{a} m_{b, a}-1} \sum_{n=0}^{k}\left(\begin{array}{l}
k \\
n
\end{array}\right) \frac{1}{k !} \\
& \cdot\left(\frac{m_{b, a} \gamma_{t h}}{\Omega_{b, a}}\right)^{k} \cdot \int_{0}^{\infty} \exp \left(-\left(\frac{m_{b, a} \gamma_{t h}}{\Omega_{b, a}}+\frac{m_{b, C}}{\Omega_{b, C}}\right) x\right) x^{n+A_{b} A_{c} m_{b, C}-1} d x \\
= & \exp \left(-\frac{m_{b, a} \gamma_{t h}}{\Omega_{b, a}}\right)\left(\frac{m_{b, C}}{\Omega_{b, C}}\right)^{A_{b} A_{C} m_{b, C}} \frac{1}{\Gamma\left(A_{b} A_{c} m_{b, C}\right)} \cdot \sum_{k=0}^{m_{b, a} A_{b} A_{a}-1} \sum_{n=0}^{k}\left(\begin{array}{l}
k \\
n
\end{array}\right) \frac{1}{k !} \\
& \cdot\left(\frac{m_{b, a} \gamma_{t h}}{\Omega_{b, a}}\right)^{k}\left(\frac{m_{b, a} \gamma_{t h}}{\Omega_{b, a}}+\frac{m_{b, C}}{\Omega_{b, C}}\right)^{-\left(n+A_{b} A_{C} m_{b, C}\right)} \cdot\left(n+A_{b} A_{C} m_{b, C}-1\right) ! .
\end{aligned}
$$

2) Relay activation probability $\mathcal{P}_{\Delta}$ :

$\mathcal{P}_{\Delta}=P_{r}\left\{\delta_{1}, \cdots, \delta_{K}\right\}$ denotes the probability that a particular relay activation sequence occurs. Whether a relay can be activated is related to the activities of those relays scheduled prior to it. Hence we can decompose $\mathcal{P}_{\Delta}$ using the chain rule $\mathcal{P}_{\Delta}=P_{r}\left\{\delta_{1}\right\} P_{r}\left\{\delta_{2} \mid \delta_{1}\right\} P_{r}\left\{\delta_{3} \mid \delta_{1}, \delta_{2}\right\} \cdots P_{r}\left\{\delta_{K} \mid \delta_{1}, \cdots, \delta_{K-1}\right\}$. In what follows, we will focus on two expressions $P_{r}\left\{\delta_{1}\right\}$ and $P_{r}\left\{\delta_{k} \mid \delta_{1}, \cdots, \delta_{k-1}\right\}$.

First, $P_{r}\left\{\delta_{1}=1\right\}$ denotes the probability that $\mathrm{R}_{1}$ can fully recover $\mathcal{I}$. This event happens only if $\mathrm{R}_{1}$ successfully decodes the signals transmitted from all the sources. Using the link decoding probability $\mathcal{P}_{b, a}^{L}(8)$, we can directly have

$$
P_{r}\left\{\delta_{1}=1\right\}=\prod_{s=1}^{M} \mathcal{P}_{\mathrm{R}_{1}, \mathrm{~S}_{s}}^{L} \text { and } P_{r}\left\{\delta_{1}=0\right\}=1-\prod_{s=1}^{M} \mathcal{P}_{\mathrm{R}_{1}, \mathrm{~S}_{s}}^{L} .
$$

To derive $P_{r}\left\{\delta_{k} \mid \delta_{1}, \cdots, \delta_{k-1}\right\}$, we define a decoding relay set $\mathcal{R}_{k-1}$ after the $(M+k-1)$ th time slot. This set contains the relays corresponding to $\delta_{i}=1$ among $\mathrm{R}_{1}, \cdots, \mathrm{R}_{k-1}$, and therefore has size $\sum_{i=1}^{k-1} \delta_{i}$. Thus $P_{r}\left\{\delta_{k}=1 \mid \delta_{1}, \cdots, \delta_{k-1}\right\}$ is the probability that $\mathrm{R}_{k}$ can correctly decode at least $M$ out of the $M+\sum_{i=1}^{k-1} \delta_{i}$ signals it received from the $M$ sources and the $\sum_{i=1}^{k-1} \delta_{i}$ relays in $\mathcal{R}_{k-1}$. We further define an $\left(M+\sum_{i=1}^{k-1} \delta_{i}\right) \times 1$ indicator vector $\kappa^{[k-1]}=\left[\kappa_{1}^{[k-1]}, \cdots, \kappa_{M+\sum_{i=1}^{k-1} \delta_{i}}^{[k-1]}\right.$, where each element $\kappa_{j}^{[k-1]} \in\{0,1\}$ denotes whether $\mathrm{R}_{k}$ can decode the $j$ th node in $\mathcal{S} \cup \mathcal{R}_{k-1}$, and $\mathcal{S}$ is the set of all sources. Now

$$
P_{r}\left\{\delta_{k}=1 \mid \delta_{1}, \cdots, \delta_{k-1}\right\}=\sum_{\sum_{j} \kappa_{j}^{[k-1]} \geq M}\left(\prod_{a_{i} \in \mathcal{S} \cup \mathcal{R}_{k-1}}\left(\mathcal{P}_{\mathrm{R}_{k}, a_{i}}^{L}\right)^{\kappa_{i}^{[k-1]}}\left(1-\mathcal{P}_{\mathrm{R}_{k}, a_{i}}^{L}\right)^{1-\kappa_{i}^{[k-1]}}\right)
$$

Again, $P_{r}\left\{\delta_{k}=0 \mid \delta_{1}, \cdots, \delta_{k-1}\right\}=1-P_{r}\left\{\delta_{k}=1 \mid \delta_{1}, \cdots, \delta_{k-1}\right\}$. Now, for each $\Delta=\left[\delta_{1}, \cdots, \delta_{K}\right]$, we substitute (9) and (10) into the chain rule to attain $\mathcal{P}_{\Delta}$.

3) Destination conditional decoding probability $\mathcal{P}_{\mathrm{D}_{n} \mid \Delta}$ : 


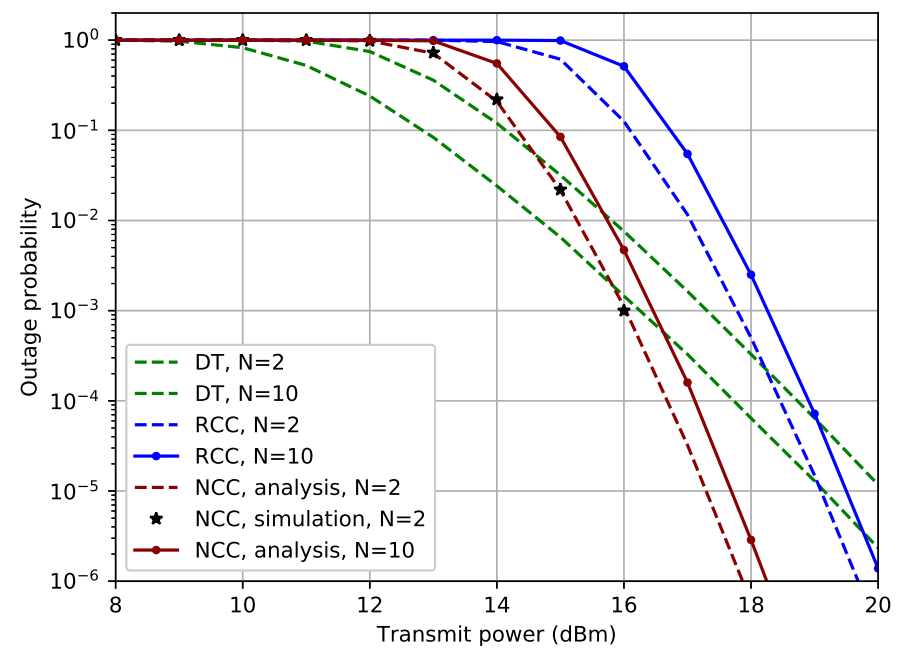

Fig. 2. System error performance comparison

We follow the logic presented in the above subsection to calculate $\mathcal{P}_{\mathrm{D}_{n} \mid \Delta}$. In particular, define $\mathcal{R}_{K}$ to be the decoding relay set after all the relays finish forwarding source messages. This set contains the relays corresponding to $\delta_{i}=1$ among $\mathrm{R}_{1}, \cdots$, $\mathrm{R}_{K}$, and has size $\sum_{i=1}^{K} \delta_{i}$. Thus $\mathcal{P}_{\mathrm{D}_{n} \mid \Delta}$ is the probability that $\mathrm{D}_{n}$ can correctly decode at least $M$ out of the $M+\sum_{i=1}^{K} \delta_{i}$, signals it received from the $M$ sources and all activated relays. Again, we define an $\left(M+\sum_{i=1}^{K} \delta_{i}\right) \times 1$ indicator vector $\pi^{[n]}=\left[\pi_{1}^{[n]}, \cdots, \pi_{M+\sum_{i=1}^{K} \delta_{i}}^{[n]}\right]$, where each element $\pi_{i}^{[n]} \in\{0,1\}$ denotes whether $\mathrm{D}_{n}$ can decode the $i$ th node in $\mathcal{S} \cup \mathcal{R}_{K}$. We have

$$
\mathcal{P}_{\mathrm{D}_{n} \mid \Delta}=\sum_{\sum_{j} \pi_{j}^{[n]} \geq M}\left(\prod_{a_{i} \in \mathcal{S} \cup \mathcal{R}_{K}}\left(\mathcal{P}_{\mathrm{D}_{n}, a_{i}}^{L}\right)^{\pi_{i}^{[n]}}\left(1-\mathcal{P}_{\mathrm{D}_{n}, a_{i}}^{L}\right)^{1-\pi_{i}^{[n]}}\right) .
$$

Substituting (10) and (11) into (4) leads to the system error probability.

Remark: The above derivation is complicated because the large-scale path loss, transmit power and antenna numbers can be different for different V2V links, which makes the decoding probability of each link unique. If the considered VCN system has the same fading and path loss factors for all V2V links (i.e., $m_{b, a}=m$ and $\Omega_{b, a}=\Omega$ ), and that between $\mathrm{C}$ and vehicles ( $m_{b, C}=m_{C}$ and $\Omega_{b, C}=\Omega_{C}$ ), and has the same transmit power and antennas at each vehicle (i.e., $P_{a}=P_{V}$ and $A_{a}=A$ ), we have the same link decoding probability $\mathcal{P}_{b, a}^{L}$ for all V2V pairs, which can be denoted by $\mathcal{P}^{L}$. We have simplified expressions

$$
\begin{gathered}
P_{r}\left\{\delta_{k}=1 \mid \delta_{1}, \cdots, \delta_{k-1}\right\}=\sum_{j=M}^{M+\sum_{i=1}^{k-1} \delta_{i}}\left(\begin{array}{c}
M+\sum_{i=1}^{k-1} \delta_{i} \\
j
\end{array}\right)\left(\mathcal{P}^{L}\right)^{j}\left(1-\mathcal{P}^{L}\right)^{M+\sum_{i=1}^{k-1} \delta_{i}-j} \\
\mathcal{P}_{\mathrm{D}_{n} \mid \Delta}=\sum_{j=M}^{M+\sum_{i=1}^{K} \delta_{i}}\left(\begin{array}{c}
M+\sum_{j=1}^{K} \delta_{i} \\
j
\end{array}\right)\left(\mathcal{P}^{L}\right)^{j}\left(1-\mathcal{P}^{L}\right)^{M+\sum_{i=1}^{K} \delta_{i}-j} .
\end{gathered}
$$

\section{NUMERICAL RESULTS}

In what follows, we use numerical results to demonstrate the advantage of the proposed scheme. We consider an example network with $M=2$ sources, $K=2$ relays and $N=2$ or $N=10$ destinations. Each node has 2 antennas. We demand all the vehicles to transmit with the same power $P_{a}$. The relationship between $P_{a}$ and $P_{C}$ is set to be $\frac{P_{a}}{P_{C}}=\tau P_{a}{ }^{\theta}=P_{a}{ }^{0.8}$, so that we can scale them together by changing $P_{a}$. The system bandwidth is set as $W=100 \mathrm{kHz}$, and the noise density is $-174 \mathrm{dBm} / \mathrm{Hz}$. The Nakagami fading parameters are set to $m_{b, a}=2, m_{b, C}=4 . \Omega_{b, a}$ and $\Omega_{b, C}$ take into account the path loss as $\Omega_{b, a}=\frac{10^{0.1 \cdot\left(1 \log _{10}\left(1000 P_{a}\right)-P L\left(d_{b, a}\right)\right)}}{1000 N_{0} A_{a}}, \Omega_{b, C}=\frac{10^{0.1 \cdot\left(10 \log _{10}\left(1000 P_{C}\right)-P L\left(d_{b, C}\right)\right)}}{1000 N_{0} A_{C}}, P L\left(d_{b, a}\right)=103.4+24.2 \log _{10} d_{b, a}$ and $P L\left(d_{b, \mathrm{C}}\right)=127+30 \log _{10} d_{b, C}$, where $d_{b, a}$ denotes the distances between a V2V pair $a$ and $b, d_{b, C}$ denotes that between $C$ and $b$, and the path loss setting follows [14] for $\mathrm{V} 2 \mathrm{~V}$ and $\mathrm{C} 2 \mathrm{~V}$ communication environments. All distances $d_{b, a}\left(d_{b, \mathrm{C}}\right)$ are generated from Gaussian distribution with mean 50m (200m) and standard deviation $2 \mathrm{~m}(5 \mathrm{~m})$.

In addition to our scheme (termed NCC, networking coding cooperation), we also consider the direct transmission (DT) and the repetition-coding based cooperation (RCC) scheme. For fair comparison, we define the average transmission rate of each scheme to be $\bar{R}=0.1 \mathrm{bps} / \mathrm{Hz}$. Fig. 2 shows that the analytical error performance of our NCC scheme is in line with results 
attained through simulation. This demonstrates the accuracy of our derivation. The DT scheme ( $R=\bar{R} M W$ bits) has lower diversity gain due to the lack of the assistance of relays (i.e., communication resource sharing in $\mathrm{VCN}$ ) to combat fading and interference. Higher power has to be consumed to guarantee sufficiently good performance. The RCC scheme attains the same diversity as NCC. But since it has to use a high data rate $(R=\bar{R} M(K+1) W$ bits) to compensate inefficient channel usage, which causes difficulties in the decoding process at the relays and destinations, especially when the received SNR is relative low. Hence the advantages of cooperative transmission emerge only when the transmit power of each node is sufficiently large. Finally, our NCC ( $R=\bar{R}(M+K) W$ bits) scheme adopts the efficient network coding concept and properly chooses the coding structure suitable for multi-source multi-relay networks. Although it uses more time slots than the DT scheme to conduct transmissions, the increase in channel consumption is limited. This fact balances the decoding errors due to fading and high data rate. The situation will become severe if more information sources and destinations are involved in the VCN, because it is easier to make errors. But high diversity still remains. These clearly show the benefits of our scheme.

\section{CONCLUSION}

We have studied applying cooperative relaying and network coding techniques to support V2V communication in VCN. We have considered a reuse-mode MIMO multi-sources, multi-relay, and multi-destination network under Nakagami-m fading. A class of MDS-FFNC is applied and the closed-form system error probability has been derived. Our results have demonstrated the potential of combining relaying and network coding techniques in future VCN.

\section{ACKNOWLEDGEMENT}

This work was funded in part by the National Natural Science Foundation of China (61771343 and 61331009), the EU PF7 QUICK project (PIRESES-GA- 2013-612652) and JSPS KAKENHI Project Number JP16H02817. This is also a part of a project that has received funding from the European Unions Horizon 2020 research and innovation programme under the Marie Sklodowska-Curie grant agreement No. 752979. R. Chen's work is supported in part by the international internship program of the National Institute of Informatics. It reflects only the authors view and the Research Executive Agency and the European Commission are not responsible for any use that may be made of the information it contains. C. Wang is the correspondence author.

\section{REFERENCES}

[1] Papadimitratos, P., Fortelle, A., Evenssen, K., Brignolo, R., Cosenza, S.: Vehicular communication systems: Enabling technologies, applications, and future outlook on intelligent transportation. IEEE Commun. Mag. 47(11), 84-95 (2009).

[2] Ahmed, E., Gharavi, H.: Cooperative vehicular networking: A survey. IEEE Trans. Intell. Transp. Syst. 19(3), 996-1014 (2018).

[3] Andrews, J.G., Buzzi, S., Choi, W., Hanly, S.V., Lozano, A., Soong, A.C.K., Zhang, J.C.: What will 5G be? IEEE J. Sel. Areas Commun. 32(6), 1065-1082 (2014).

[4] Lee, E., Lee, E.K., Gerla, M., Oh, S.Y.: Vehicular cloud networking: Architecture and design principles. IEEE Commun. Mag. 52(2), 148-155 (2014).

[5] Whaiduzzaman, M., Sookhak, M., Gani, A., Buyya, R.: A survey on vehicular cloud computing. Journal of Network and Computer Applications 40, 325 -344 (2014)

[6] Kim, S.W., Qin, B., Chong, Z.J., Shen, X., Liu, W., Ang, M.H., Frazzoli, E., Rus, D.: Multivehicle cooperative driving using cooperative perception: Design and experimental validation. IEEE Trans. Intell. Transp. Syst. 16(2), 663-680 (2015).

[7] Dolk, V., d. Ouden, J., Steeghs, S., Devanesan, J.G., Badshah, I., Sudhakaran, A., Elferink, K., Chakraborty, D.: Cooperative automated driving for various traffic scenarios: Experimental validation in the GCDC 2016. IEEE Trans. Intell. Transp. Syst. 19(4), 1308-1321 (2018).

[8] Li, M., Yang, Z., Lou, W.: CodeOn: Cooperative popular content distribution for vehicular networks using symbol level network coding. IEEE J. Sel. Areas Commun. 29(1), 223-235 (2011).

[9] Xiao, M., Kliewer, J., Skoglund, M.: Design of network codes for multiple-user multiple-relay wireless networks. IEEE Trans. Commun. 60(12), 3755-3766 (2012).

[10] Wang, C., Xiao, M., Skoglund, M.: Diversity-multiplexing tradeoff analysis of coded multi-user relay networks. IEEE Trans. Commun. 59(7), 1995-2005 (2011).

[11] Xing, W., Liu, F., Wang, C., Xiao, M., Wang, P.: Multi-source network-coded D2D cooperative content distribution systems. Journal of Communications and Networks 20(1), 69-84 (2018).

[12] Soleimani-Nasab, E., Matthaiou, M., Ardebilipour, M.: Multi-relay MIMO systems with OSTBC over Nakagami- $m$ fading channels. IEEE Trans. Veh. Technol. 62(8), 3721-3736 (2013).

[13] Chalise, B.K., Czylwik, A.: Exact outage probability analysis for a multiuser MIMO wireless communication system with space-time block coding IEEE Trans. Veh. Technol. 57(3), 1502-1512 (2008).

[14] 3GPP TR 36.814: Further advancements for E-UTRA physical layer aspects. (2009) 